\title{
THERE IS A BEEP
}

\section{BY FILIP WILTGREN}

There is a beep.

An applied neurophysicist walks into a bar. It's a nice bar, red leather upholstery, jukebox in the corner, bartender in a white dinner jacket. More of a lounge, really. And this neurophysicist walks into the place like he owns it.

Maybe he does.

"Joe," he says, sitting down, "fix me up a stiff one."

And the bartender bends behind the bar and comes up with one of these silver shakers that rattle like a million dollars on ice and he rattles it and breaks it and there's this rich brown, velvety fluid emerging from the shaker and sloshing in slow motion into a heavy, cutcrystal glass.

The physicist goes to the lavatory. You know it's the lavatory because it says lavatory right on the door. Not toilet, not WC. Lavatory.

That's because the physicist is British and hasn't taken to the language of wherever it is he is.

So now the neurophysicist is gone. The lavatory door swings shut behind him.

\section{There is a beep.}

A soon-to-be-Nobel-laureate neurophysicist walks into a bar. It's a nice bar, the kind with red leather upholstery with big round buttons. Sinatra's crooning from a jukebox hooked into a Bang \& Olufsen sound system. The bartender looks classy, like he makes more in an hour than most chief executives.

"Joe," says the physicist, "fix me a stiff one."

And the bartender does his magic, and the liquid flows and sloshes, and there's a smell like old oak and cinnamon. Beads of dew condense on the glass and flow down to the physicist's hand. He lifts the glass to his mouth, tilts it. It tastes like victory. The neurophysicist smacks his lips in appreciation. He raises his finger to order another but then decides against it. He pulls out a billfold and removes an Irish $£ 10$ note. James Joyce winks at him from the paper. That feels wrong, somehow.

The physicist walks to the door, but it's locked. He gets a slightly worried look on

\section{One-way traffic.}

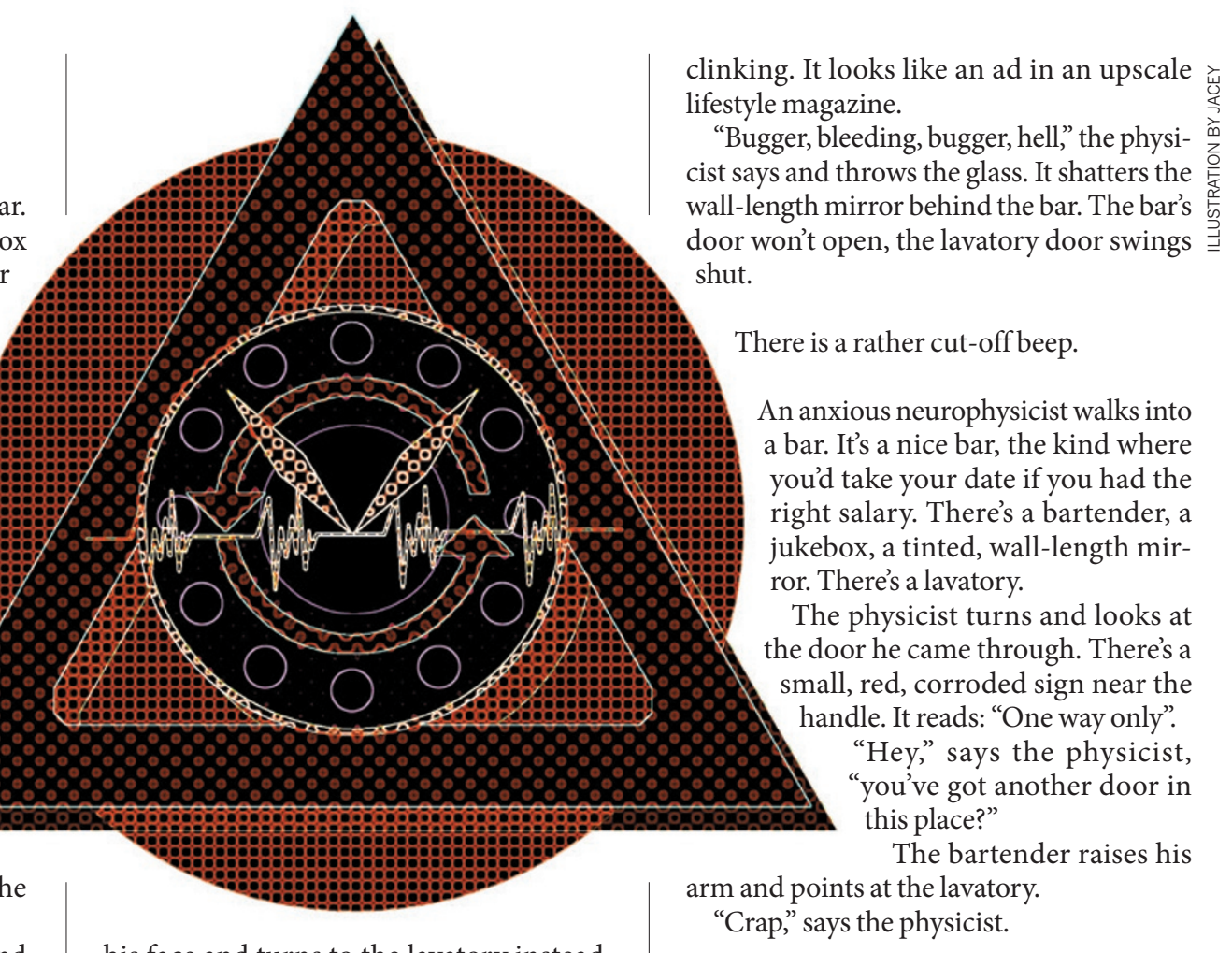

his face and turns to the lavatory instead. The lavatory door swings shut.

There is another beep.

A somewhat confounded neurophysicist walks into a bar. It's a nice bar, retro styled with lacquered benches the colour of maraschino cherries. There's a mahogany bar, a balding bartender, a jukebox playing 'Auld Lang Syne' in the background.

"Hey," says the physicist, "fix me one of those."

He points to a row of bottles standing at attention on a shelf behind the bar. They're all Bushmills 21-year-old single malt. Every second one is half empty.

The bartender bends down and hauls out a new bottle of malt. The seal cracks like fireworks. The liquid sloshes in slow motion into a glass full of ice cubes, condensation forming on the outside. A single drop runs down the side of the glass, amplifying the perfect, lip-smacking clarity of the liquid behind it. The physicist picks up the glass.

It feels completely dry, like paper. $\rightarrow$ NATURE.COM

Follow Futures: y @NatureFutures f go.nature.com/mtoodm
The physicist tilts the glass. The whiskey stays the same, frozen in a half swirl, the ice cubes almost
There is no beep.

There's this bar, and there's a physicist in it, and the bar's got two doors, one that opens and one that don't. The one that opens is marked "Crapper".

The physicist is confused. He doesn't need to go to the crapper, he needs a drink and he doesn't know why he needs it.

"Hey," he says to the bartender, "fix me up a shot, will ya?"

The bartender bends down behind a row of antique silver drink shakers and pulls out a shotgun. It goes well with the tweed cap he's got on. He points it at the physicist.

"Crap," he says and pulls the trigger.

The leather upholstery is decorated in splattered-neurophysicist chic.

There is no beep, there is no bar, there is no neurophysicist. There is only a failed experiment, a final ECG tone, a long, drawn-out, unpleasant investigation, and a case study on the importance of following established safety protocols.

Filip Wiltgren is a communications officer at Linköping University by day and a writer, game designer and worrier by night. He lives in Sweden with his wife and daughters. 